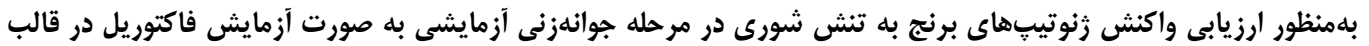

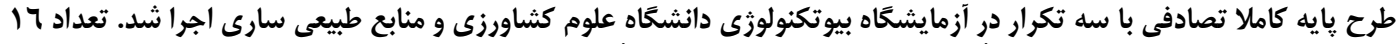

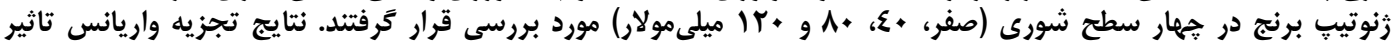

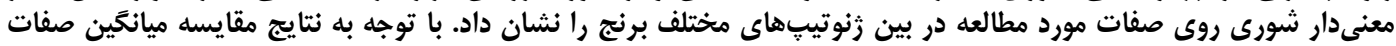

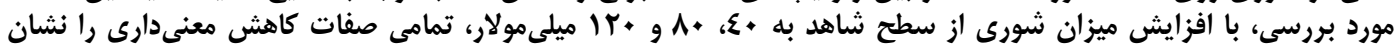

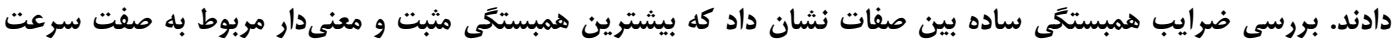

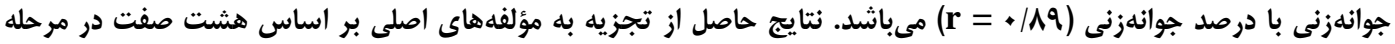

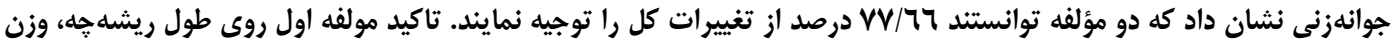

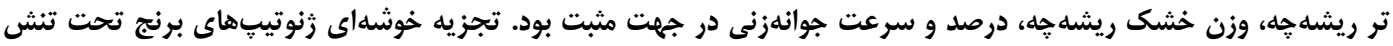

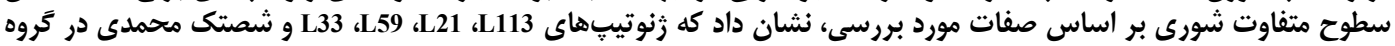

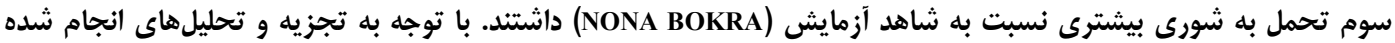

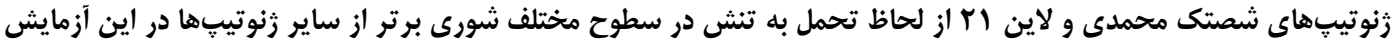

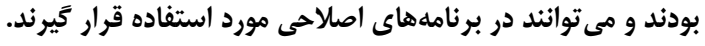

وازههاى كليدى: برنج، تنش شورى، جوانه زنى، تجزيه خوشهاى، تجزيه به مولفههاى اصلى

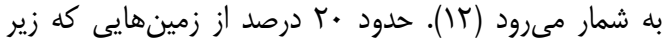

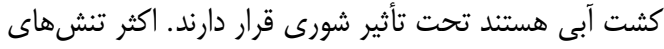

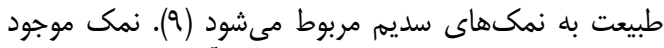

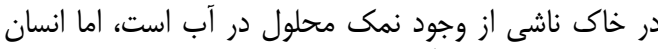

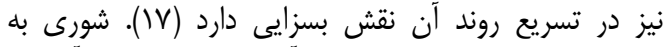

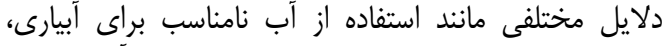

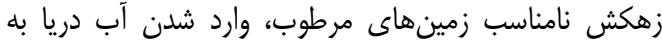

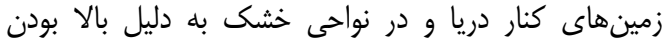

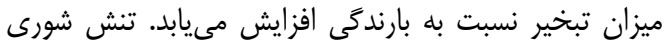

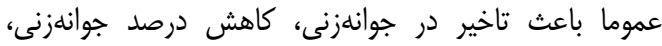

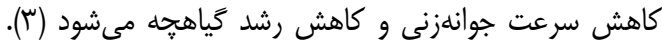

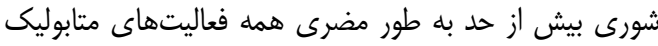

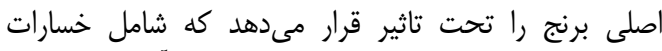

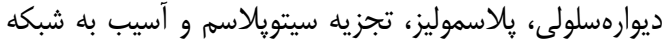

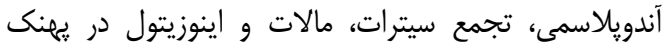

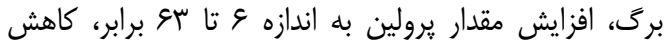

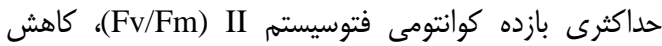

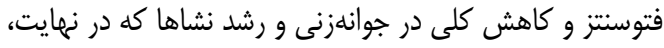

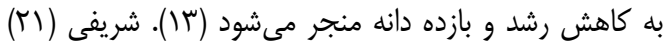

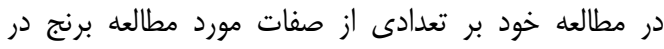

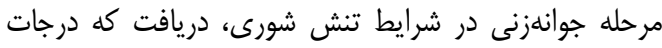

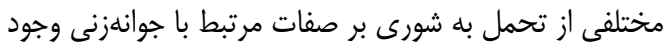

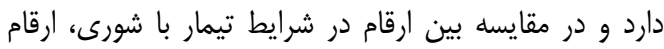

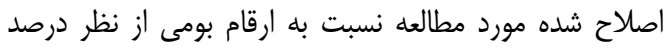

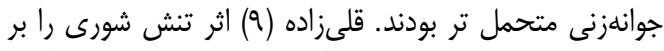

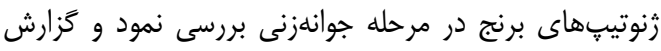

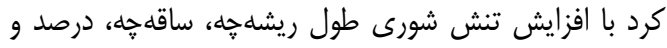

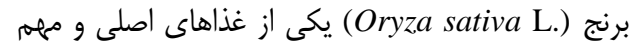

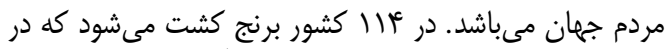

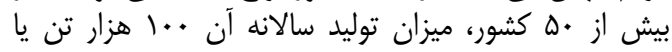

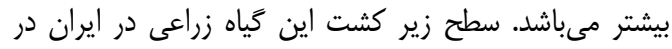

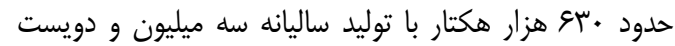

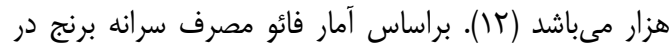

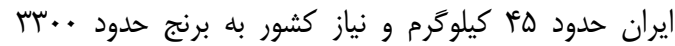

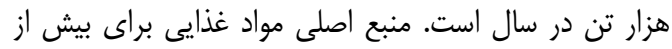

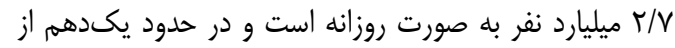

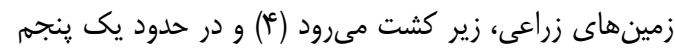

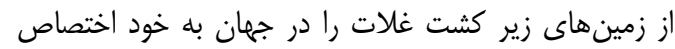

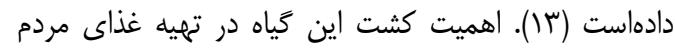

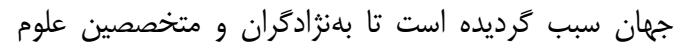

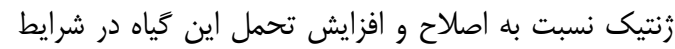

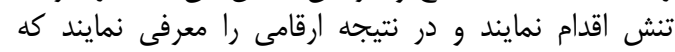

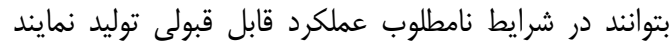
مي

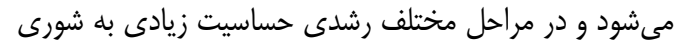

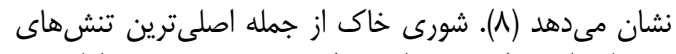

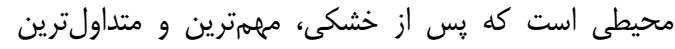

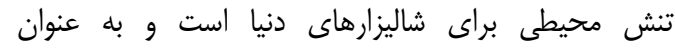

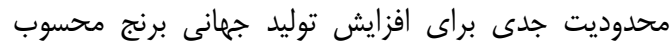

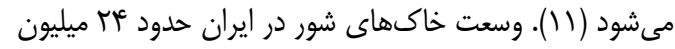

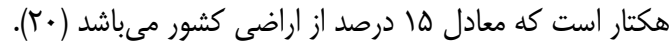

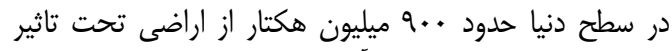

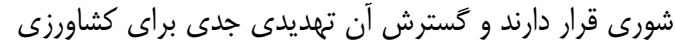




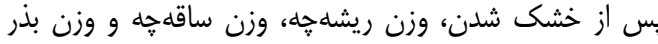

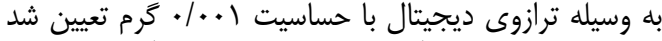

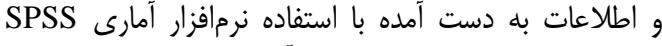

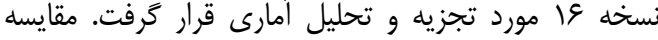

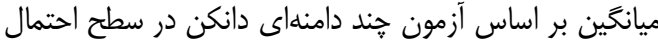
ه درصد انجام شد. از تبديل دادئ داده

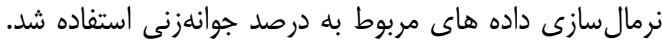

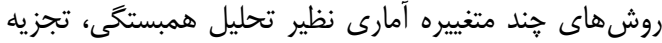

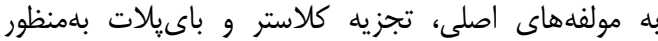

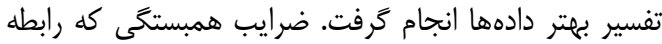

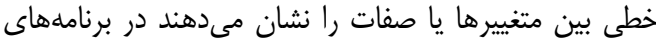

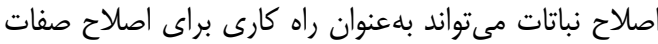

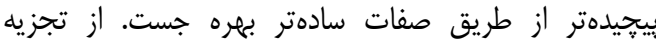

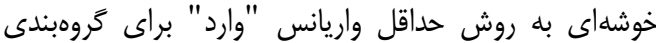

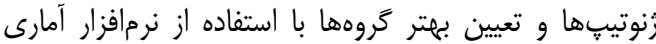
SPSS

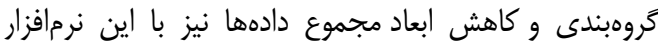

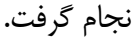

\section{نتايج و بحث}

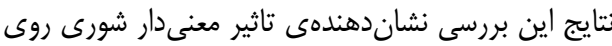

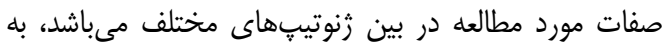

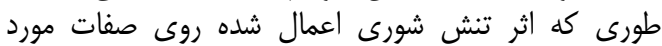

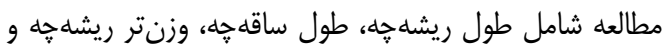

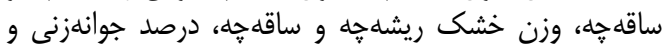

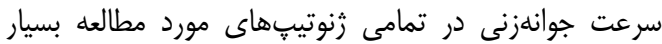

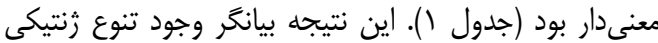

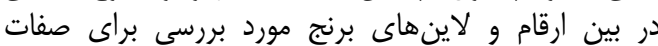

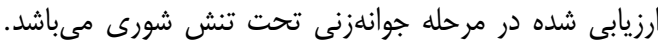

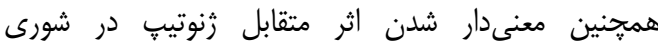

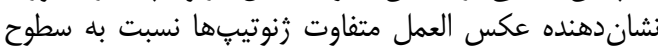
متفاوت تنش شورى مئباشد.

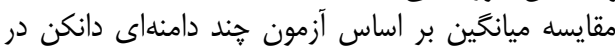

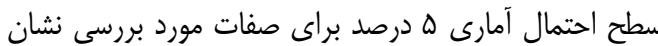

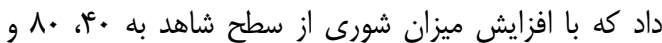

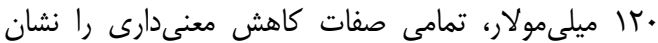

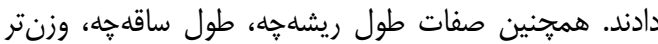

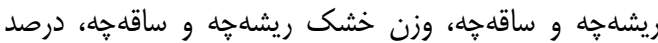

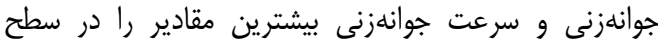

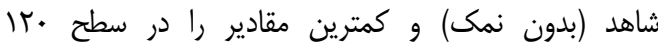

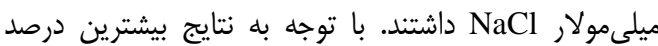

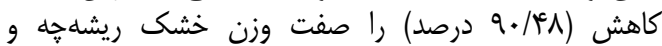

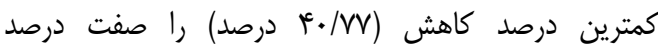

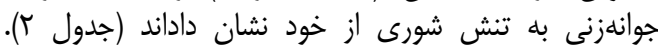

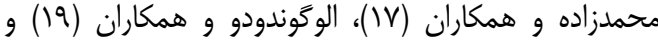

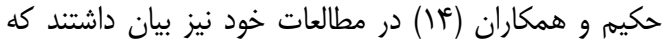
افزايش سطوح شورى باعث كاهش صفات جوانهزنى در برنج مىشود.
سرعت جوانهزنى كاهش معنىدارى ييدا كرد. محمدزاده و

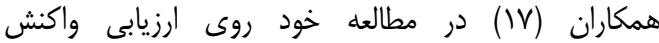

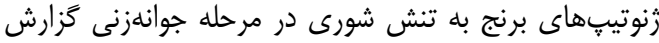

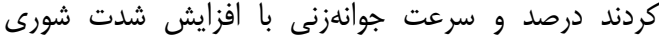

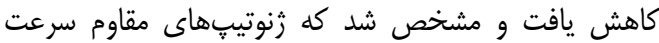

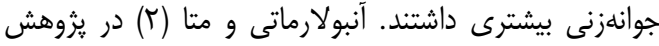

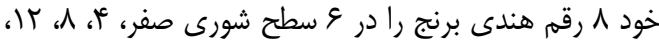

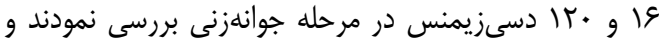

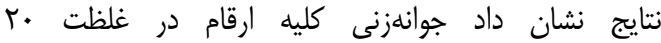
دسىزيمنس متوقف شد همجنين با افزايش سطوح شورى إنى

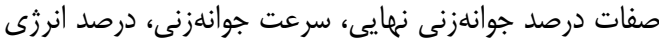

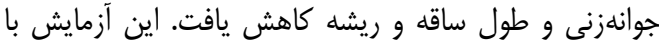

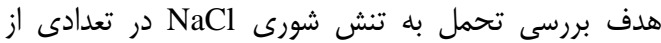
لاينهاى اميد بخش برنج در در مرحله جوانهزنى انجام كرديد.

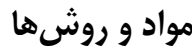

بلهنظور بررسى اثر تنش شورى در در مرحله جوانهزنى برخى

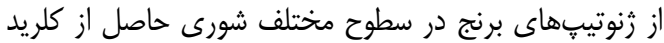

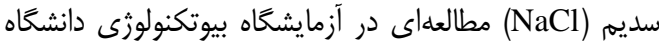

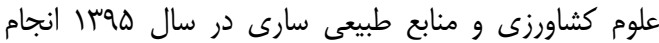

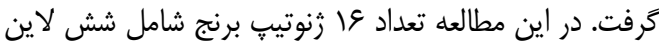

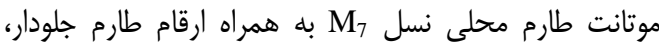

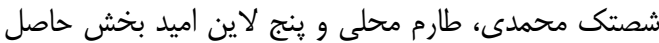

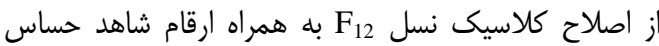

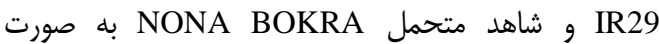
آزمايش فاكتوريل در قالب طرح پايه كاملا تصادفى با سه

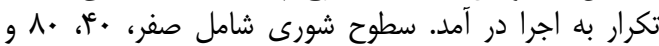

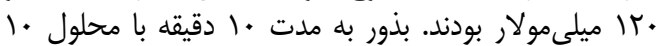

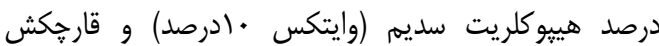

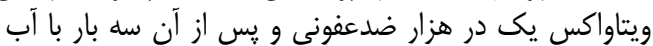

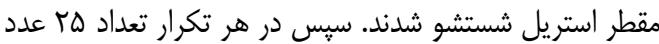

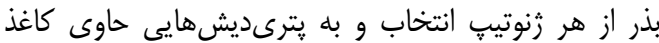

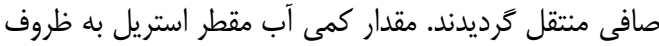

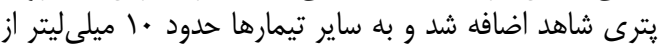

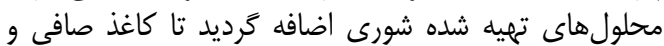

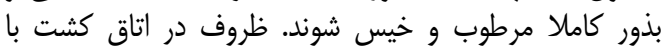

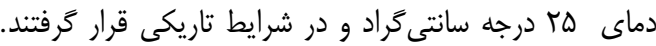

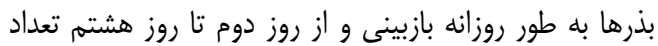

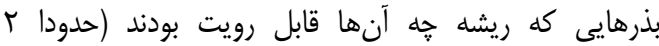

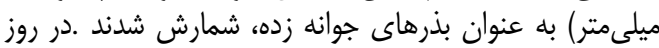

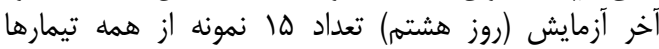

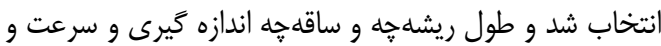

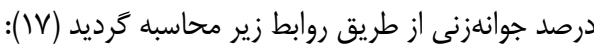
(n $=\left(\mathrm{n}_{\mathrm{i}} / \mathrm{N}\right) \times 1 \ldots$

n $=n_{1} / D_{1}+n_{2} / D_{2}+000+n_{i} / D_{i}$

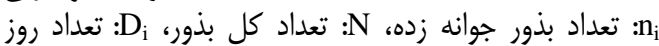

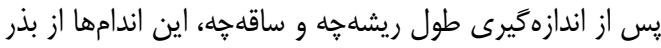

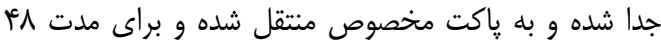

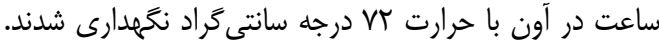



ارزيابى واكنش برخى زنوتيڤهاى برنج به تنش شورى در مرحله جوانهزنى

Table 1. Analysis of variance for measured characteristics

جدول ا- تجزيه واريانس خصوصيات اندازه گيرى شده

\begin{tabular}{|c|c|c|c|c|c|c|c|c|c|}
\hline \multirow[b]{2}{*}{ درصد جوانهزنى } & \multicolumn{5}{|c|}{ ميانگين مربعات } & \multirow[b]{2}{*}{ 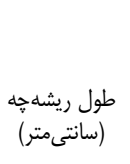 } & \multirow[b]{2}{*}{ 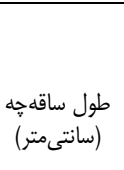 } & \multirow[t]{2}{*}{ آزادى } & \multirow[t]{2}{*}{ منابع تغييرات } \\
\hline & جوانهزنى & وزئن خهيه & وزن ساقه خشه & 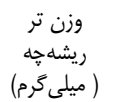 & 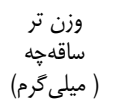 & & & & \\
\hline $1 \cdot 9 \cdot r / r q * *$ & $f \cdot N / / r^{* * *}$ & $\cdot / \cdot \cdot r^{\mu * * *}$ & . $/ . \mu^{\mu * *}$ & . $/ . \mathrm{rT}^{* * *}$ &.$/ .4 \psi^{* * *}$ & $\uparrow V / \cdot \Gamma \omega^{* * *}$ & $F \backslash / \Gamma^{* * *}$ & $r$ & شورى \\
\hline$\Lambda \varepsilon \cdot / \Delta \Delta^{* *}$ & $\Gamma \Delta \Gamma / \Delta \Delta_{* *}$ & $\cdot\left(\ldots,\left.\right|^{* * *}\right.$ & $\left.\cdot|\ldots|\right|^{* * *}$ & $\% \Delta^{* *}$ &.$/ . \gamma^{* * *}$ & $\mid \Gamma / F \uparrow * *$ & $\mid / 91 * *$ & 10 & رنوتيب \\
\hline$r \cdot . / F V^{* * *}$ & $\mid V / \Delta \Gamma^{* * *}$ & $\cdot|\ldots| \cdot \mid * *$ & $\circ / \ldots . \Delta ৭ * *$ &.$/ . r^{* * *}$ &.$/ . . \mu * *$ & $r / \Gamma \mid * *$ & $\cdot \mid 94 * *$ & is & رنوتيب x \\
\hline$\Delta / M$ & $\cdot / F \cdot r$ & $\cdot 1 \cdots \cdot v$ & $\cdot / \ldots \ldots+t$ & $.1+.1$ &.$\ldots r$ & $\cdot / 1 \cdot 1$ & $.1 \cdot 4 t$ & $1 T \Lambda$ & خطا آزَمايشى \\
\hline$r / \Delta r$ & $\Delta / \Delta S$ & $I T / v g$ & $10 / \mp \Lambda$ & $1 . / \Lambda F$ & N/9V & $N / N r$ & 1.194 & & ضريب تغييرات \\
\hline
\end{tabular}

"

جدول r- مقايسه ميانكَين خصوصيات اندازمكيرى شده در سطوح مختلف شورى در در آزمايش جوانهزنى Table 2. Mean comparison of the measured characteristics in different levels of salinity in germination experiment

\begin{tabular}{|c|c|c|c|c|c|c|c|c|}
\hline جوانهزنى درص & جوانهزنى & وزئن خشهى & وزن خاقه خهى & (ميلى رَّه & (ماقه & طول ريشه خه & طول ساقه خهـ & 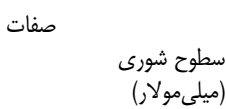 \\
\hline$\Lambda V / \Delta \Lambda^{a}$ & $1 r / a r^{a}$ & $r / *^{a}$ & $r \cdot 1^{a}$ & sV/Tra & $q \cdot / \Delta q^{a}$ & $r / r q^{a}$ & $r / q r^{a}$ & صفر \\
\hline$\checkmark / \wedge r^{D}$ & $1 r / \kappa^{c} \cdot 9^{D}$ & $\mid f / .^{\mathrm{D}}$ & $19 / 0^{0}$ & Q) $/ 9 \cdot{ }^{\circ}$ & $\Lambda \varepsilon / \Gamma \Delta^{D}$ & $F / T \Delta^{D}$ & $r / F \Lambda^{D}$ & f. \\
\hline $1 V / 91$ & $r / \varnothing V$ & FT/NE & $\Delta$ & Tr/A & $F / \varnothing \Delta$ & $\mathrm{T} / \mathrm{IV}$ & $|Q| \cdot V$ & درصد كاهش \\
\hline$q \mu / \cdot \varphi^{c}$ & $1 \cdot / \Delta 9^{c}$ & $\mathrm{~N} / \cdot^{\mathrm{c}}$ & $\mid r / 0^{c}$ & $f \cdot / V \mu^{c}$ & $\Delta N / T)^{c}$ & $9 \Lambda \mu /^{c}$ & $1 / V 10^{c}$ & $\wedge$ \\
\hline $\mathrm{rN} \cdot \mathrm{r}$ & rarar & 9)/9. & f. & rq/f & $r \Delta / v r$ & $18 / 18$ & fi/tr & درصد كاهش \\
\hline$\Delta U / A V^{a}$ & $V / \Delta q^{a}$ & $r / *^{a}$ & $F / 0^{a}$ & $1 \varepsilon / 10^{a}$ & $r \Delta / V \omega^{a}$ & $r T \Delta r /^{a}$ & $\cdot / \Lambda \cdot \Delta^{a}$ & $\pi$ \\
\hline $\mathrm{r} \cdot / \mathrm{VV}$ & $F Q / F V$ & $q \cdot / 4 \wedge$ & $\wedge$. & $\mathrm{VQ} / \mathrm{Q \Lambda}$ & $V / \Delta V$ & eq/ru & $V T / F T$ & درصد كاهش \\
\hline
\end{tabular}

ويزه يونهاى سديم و كلر نيز با تاثير منفى بر مراحل تقسم

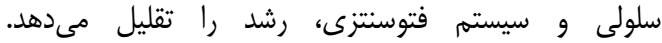
زنوتيڤهاى شصتك محمدى، M181 و

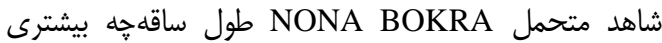

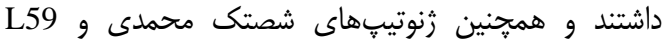

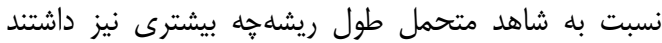

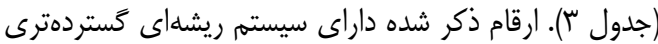

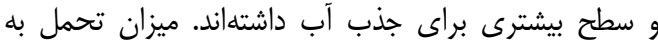

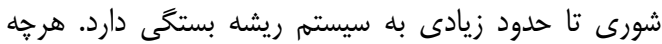

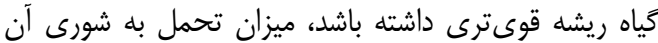

بيشتر است (1) بئه

وزن تر ساقهجه و ريشه

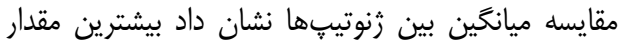

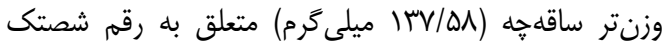

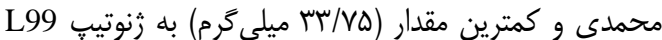

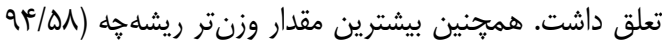

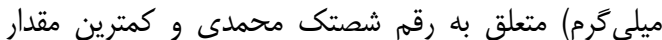

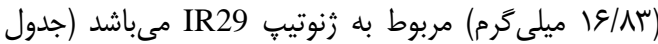

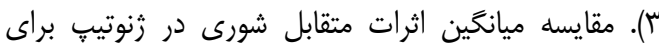

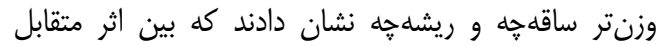

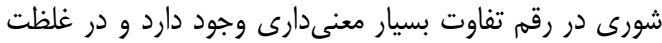

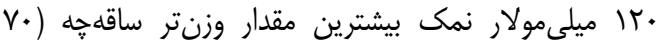

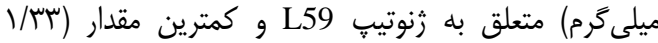

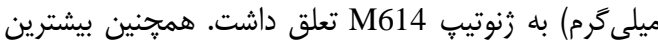

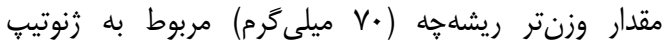
وONA BOKRA
طول ساقه ֶِه و ريشه

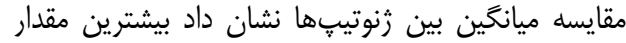

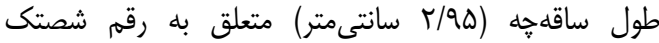

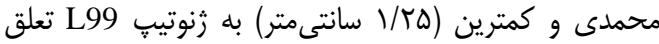

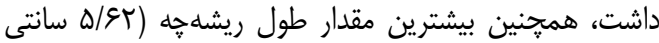

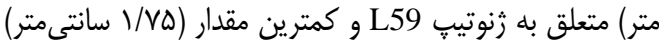

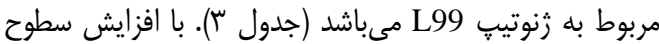

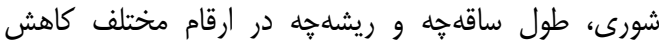
يافت، به طورى كه بيشترين طول ساقهجه (ه سانتى

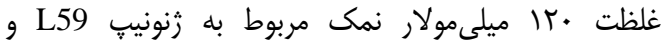

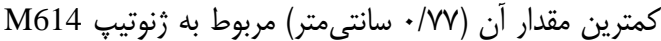

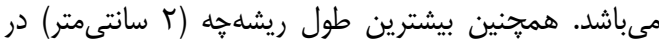

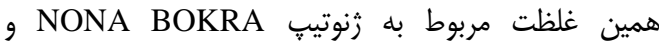

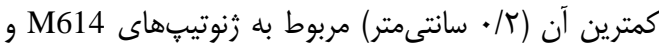

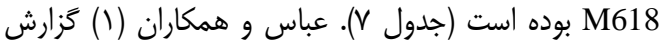

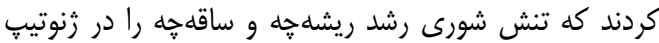

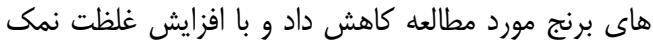

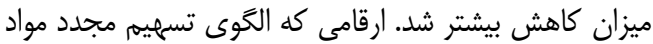

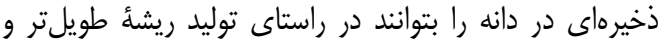

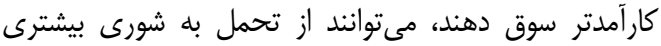

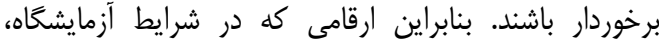

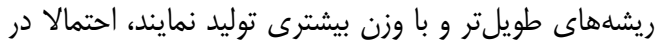

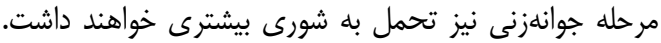

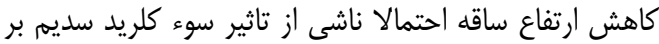

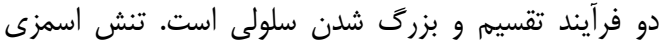

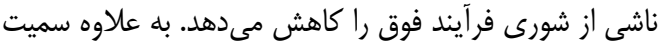




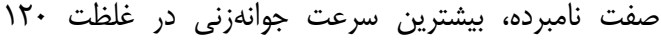

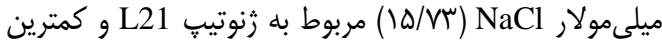

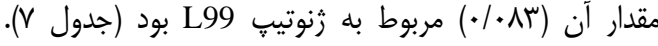

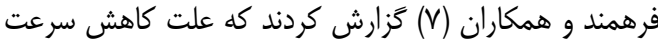

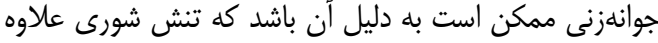

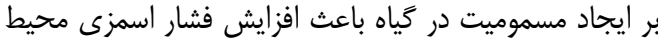

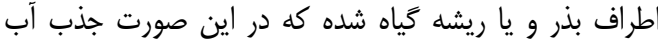

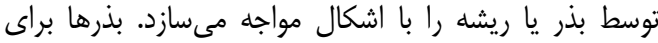

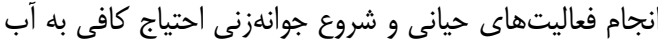

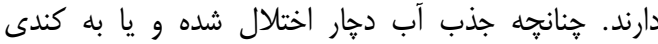

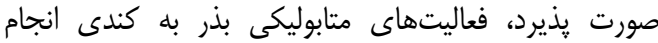

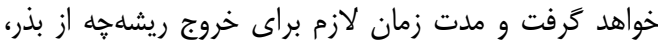

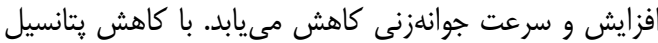

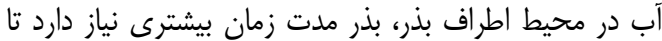

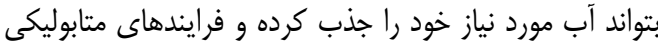

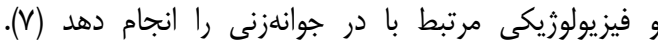

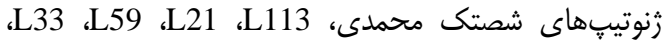

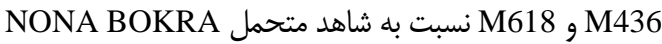
سرعت جوانهزنى بيشترى داشتند (جدول بَّ). شورى باعث ماند

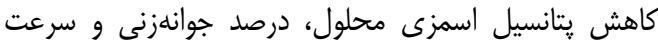

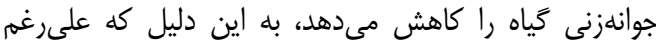

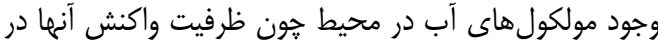

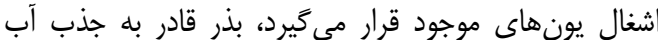

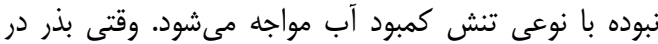

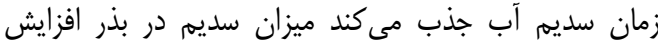

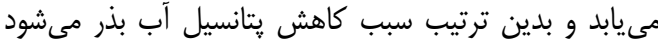

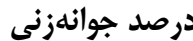

مقايسه ميانگين بين زنوانيقها

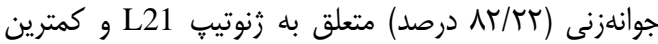

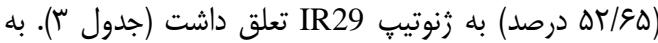

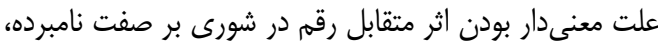

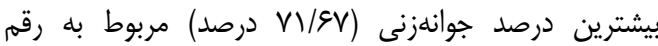

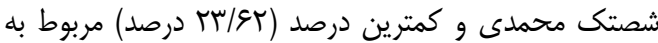

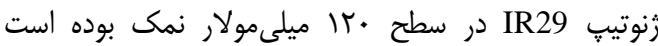

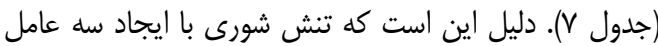

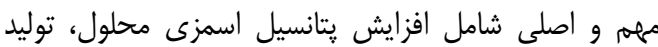

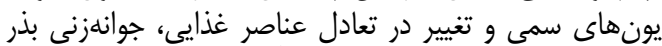

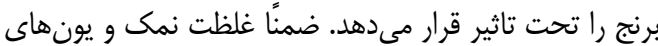

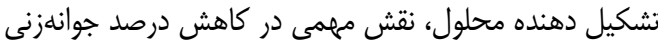

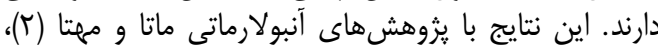

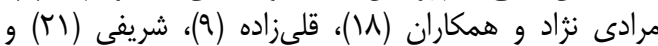

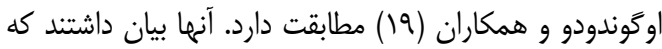

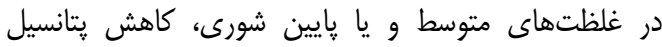

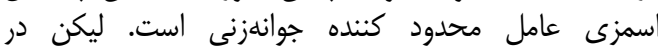

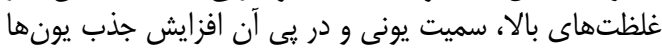
به خصوص يون

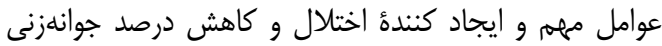

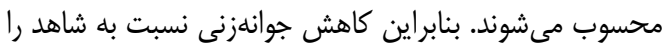

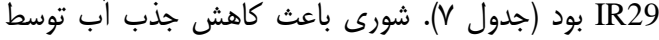

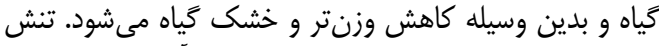

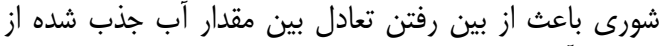

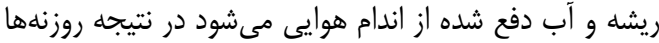

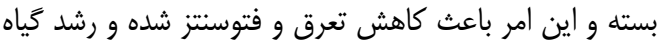

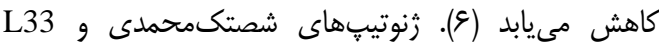

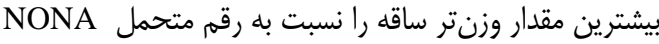

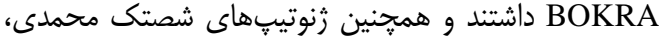

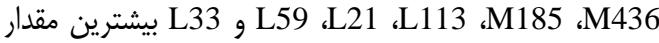

وزنتر ريشه קه را نسبت به شاهد متحمل داشتند (جدول سا).

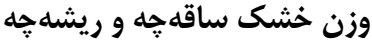

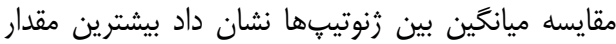

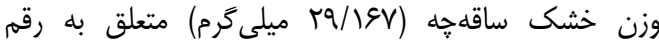

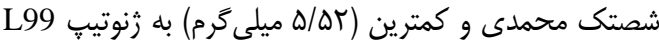

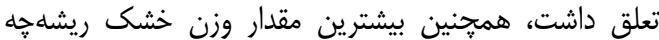

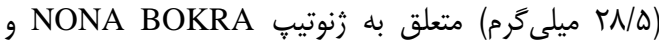

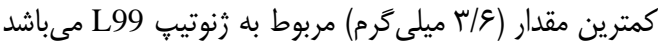

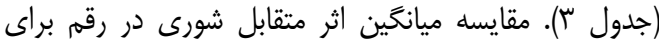

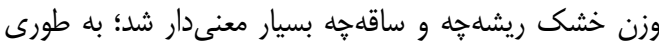

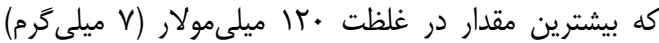

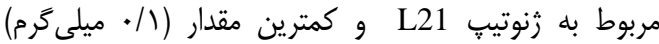

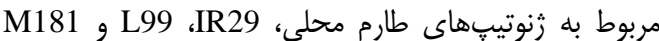

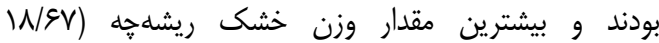
ميلى

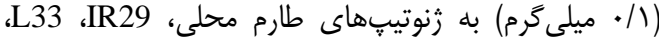

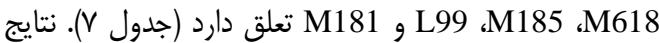

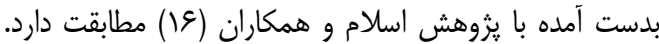

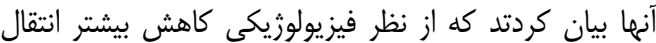

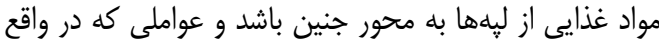

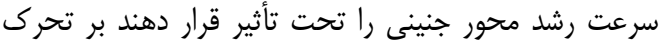

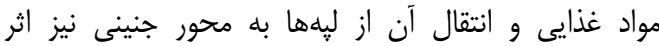

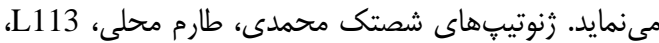

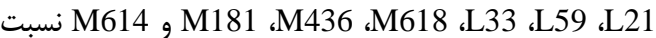

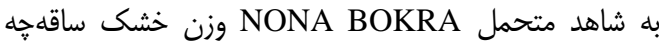

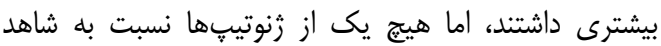

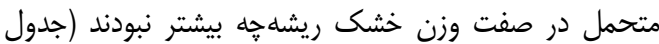

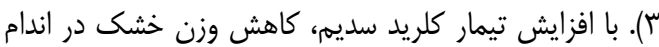

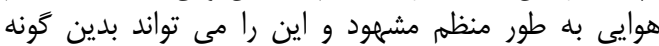

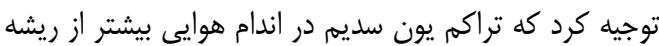

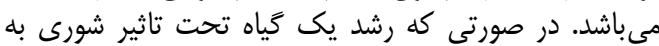

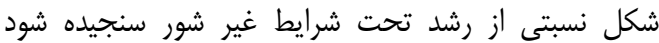

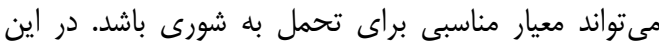

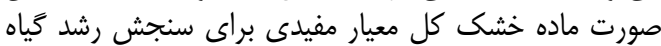

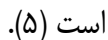

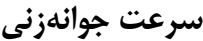

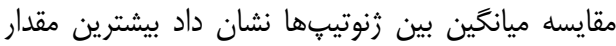

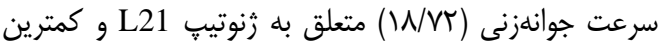

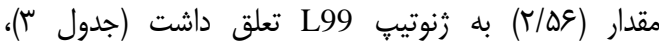
همجنين به علت معنى دار بودن اثر متقابل رقم در شورى بر 
L33 نسبت به شاهد متحمل درصد جوانهزنى بيشترى داشتند

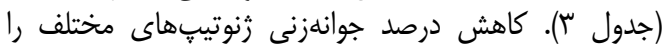

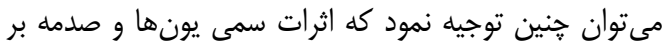

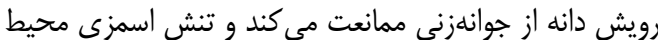

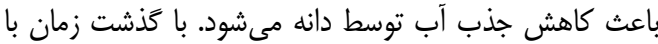

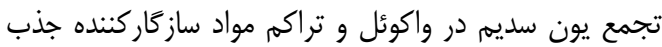

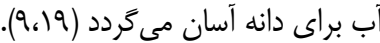

مىتوان به اثرات يونى نمك و همينطور كاهش يتانسيل آب،

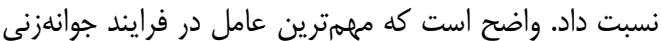

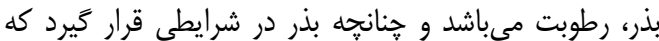

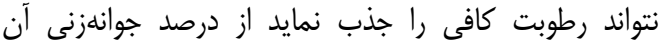

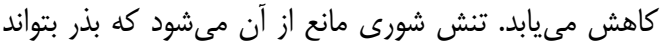

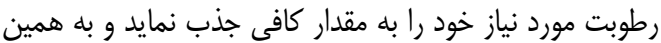

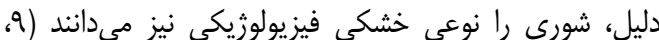

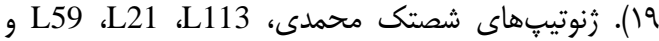

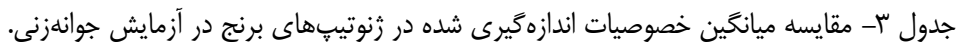
Table 3. Mean comparison of the measured characteristics in rice genotypes in germination experiment.

\begin{tabular}{|c|c|c|c|c|c|c|c|c|}
\hline جوانهزنى درصد & سوانهزنى & وزن ريشه خشه & 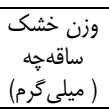 & وزن (ميلى رَّم) & وزنتر ساقه خه & طول ريشه جه (سانتىمتر) & طول ساقه & صفان \\
\hline $\mathrm{V} / \mathrm{VV} \mathrm{v}^{\mathrm{D}}$ & $1 Q / \& Y^{C}$ & 19/r" & $r q / 1 \notin v^{a}$ & $q 4 / \Delta \Lambda^{a}$ & $\| m v / \Delta \Lambda^{a}$ & D/T & $r / 9 \Delta^{a}$ & شصتك محمدى \\
\hline $94 / 9 q^{n}$ & 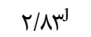 & $9 / .19 n$ & $11 / \Delta^{g}$ & TE/KF' & 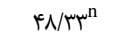 & $\mathrm{f} / \mathrm{q}^{\mathrm{e}}$ & $1 / / V^{g}$ & طارم جلودار \\
\hline $9 N / 99^{\text {eI }}$ & IIITI & $N / F^{e} e^{\operatorname{Ign}}$ & $1 / / q 8^{D}$ & $\mathrm{rq} / \Delta .^{e}$ & $v^{c} / / v^{a}$ & $r / Q \Lambda^{g}$ & $1 / 9 \mu^{a e r}$ & طارم محلى \\
\hline$\Lambda r / r^{\mathrm{a}}$ & $M / V Y^{a}$ & $I T / r \Delta^{c}$ & & $\Delta T / T \omega^{c a}$ & $9 \cdot 1 \cdot 1^{\mathrm{eI}}$ & $r / q^{\text {ae }}$ & $1 / \Lambda \varepsilon \Delta^{e r g}$ & لاين آ \\
\hline$V \Delta / T V^{c}$ & $|\omega / F V|^{c}$ & $1 \cdot / V \Delta^{\mathrm{ae}}$ & $1 \% / \Delta^{\text {aerg }}$ & $\mathrm{FV} / .^{\mathrm{ae}}$ & $\mathrm{FV} / \mathrm{IV} \mathrm{V}^{\mathrm{n}}$ & $\Delta / \&)^{\mathrm{a}}$ & 1/querg & لآين ه9 \\
\hline$\Delta T / \& C^{J}$ & $r / \Delta \omega^{J}$ & $r / \varphi^{1}$ & $\Delta / \Delta Y^{n}$ & $r \mu / V Q^{1 g}$ & سM/Vag & $1 / V \Delta^{1}$ & $1 / T \Delta^{1}$ & لان \\
\hline$V \Psi / r V^{a}$ & $\mid F / r \xi^{a}$ & Q/. Freag & $|Q / 9|^{c}$ & $\Delta V / q Y^{c}$ & $v \mu / \cdot \Lambda^{a}$ & $r / \cdot \Lambda^{\text {ae }}$ & $1 / \Lambda \mu^{e r g}$ & لاين س" \\
\hline$\Lambda \cdot / 9 v^{a}$ & $I V / F D^{D}$ & $19 / \Delta^{D}$ & $\mid \alpha / \cdot 1^{\mathrm{ca}}$ & D ס V V & $\Lambda 9 / r \Delta^{D}$ & $r / \iota^{g}$ & $1 / \Delta q^{n}$ & لاين سا| \\
\hline$\Delta T / \& \Delta^{J}$ & $8 / \cdot 1^{1}$ & V/Trg & $V / \mathrm{r}^{\mathrm{n}}$ & I $8 / \wedge \mu^{g}$ & $M N / \Delta \Lambda^{1}$ & $r / V^{e^{g n}}$ & $1 / \Delta r^{n}$ & IR29 \\
\hline$V \cdot / 4 r^{e}$ & $|1 / 8|^{\mathrm{eI}}$ & $r N / \Delta^{a}$ & $|r /| q^{\mathrm{Ig}}$ & 舟 & $\Lambda \cdot 1^{\mathrm{c}}$ & $\Gamma / \Delta^{c}$ & $T / T \mathcal{G}^{\mathrm{Dc}}$ & NONA BOKRA \\
\hline $9 V / q^{1 g}$ & $1 \cdot 1 \cdot e^{\mathrm{n}}$ & $11 / v \Delta^{\text {cae }}$ & $I r / V V^{\text {erg }}$ & $r V / \Delta \Lambda^{I}$ & $\Delta V / q V^{I}$ & $r / T v^{c}$ & $1 / 9 \vee \Delta^{a e r}$ & M614 \\
\hline $9 V / \gamma^{I g}$ & $11 / q \mathrm{~V}^{\mathrm{eI}}$ & $N / 19^{g n}$ & $1 \Psi / \Delta^{\text {aerg }}$ & $\mu \cdot / T \Delta^{I}$ & $\Delta r / \cdot \Lambda^{g n}$ & $r / \Lambda)^{g n}$ & $\mid / \wedge)^{\mathrm{Ig}}$ & M618 \\
\hline $9 \Delta / / \Gamma^{n}$ & $1 \cdot / \Delta)^{\mathrm{gn}}$ & s/sqg & $\mid r / r v^{\text {aerg }}$ & $r T / r Q^{I g}$ & $q \mu / r \Delta^{e}$ & $r / V \Delta^{g n}$ & $T / F \cdot \Delta^{D}$ & M181 \\
\hline ا & $11 / \xi^{\mathrm{eI}}$ & $V / \Delta \Lambda^{g n}$ & $r \cdot / 1 \varepsilon^{D}$ & $\Delta r / \Delta V^{c a}$ & $V \notin / \Lambda \mu^{a}$ & $r / r q^{I}$ & $r / r V^{D}$ & M184 \\
\hline$g q / \psi^{g n}$ & $1 \cdot / \mathrm{V}^{\mathrm{g}}$ & $11 / \Gamma \Delta^{\text {cae }}$ & $11 / 89^{g}$ & $\kappa q / \Lambda \mu^{c a}$ & $\Delta F / \kappa^{\prime r g}$ & $r / \Delta F^{n}$ & T/Ifrca & M185 \\
\hline $99 / 9 V^{e}$ & $11 / \wedge r^{e}$ & $|r / q|^{\mathrm{ca}}$ & $\mid f / \Delta \Lambda^{\mathrm{cae}}$ & fV/fre & $V \Delta / T \Delta^{\mathrm{I}}$ & $F / \mu^{c a}$ & $r / \cdot 1 \Delta^{\mathrm{ae}}$ & M436 \\
\hline
\end{tabular}

جدول عأ- مقايسه ميانگين زنوتيڤهاى برنج براى صفات جوانهزنى در سطح شاهد Table 4. Mean comparison of rice genotypes for germination traits in normal level

\begin{tabular}{|c|c|c|c|c|c|c|c|c|}
\hline جوانهزنى & جوانهزنى & وزيله خها & وز ساقه خشه & (ميلى & 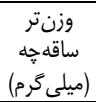 & 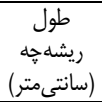 & 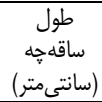 & صفات \\
\hline$A V / R r^{\prime \prime}$ & $r . / 1 I^{3}$ & $\mathrm{TV} / \mathrm{o}^{\circ}$ & $\mathrm{rN} /$ & $\mid V Q / 0^{*}$ & $\mid V \Delta / r T^{\prime \prime}$ & $9 / 99^{\circ}$ & $r / V r^{\prime \prime}$ & شصتك محمدى \\
\hline$\Lambda F / g V^{*}$ & $g / 4 \cdot r^{*}$ & | & $\mathrm{IV} / \mathrm{.}$ & $F \Delta /$. & $v e / . "$ & $\Delta / \Delta)$ & $r / T V^{*}$ & طارم جلودار \\
\hline$\wedge \mathrm{V} /$. & $\mid r / \Delta r$ & $181 . *$ & $1 F / \mu^{*}$ & $r .1 . *$ & $11 \% \% *$ & $r / \mu \psi^{*}$ & $r / q V^{*}$ & طارم محلى \\
\hline$M N / \wedge 9^{\circ}$ & $r \cdot / q r^{*}$ & $r r / . *$ & $\mid 9 / 9 V^{*}$ & $\Lambda F / \mu^{*}$ & $1.910^{*}$ & $F / Q^{*}$ & $r / \Lambda r^{* \prime \prime}$ & لاين /r \\
\hline M/ " & 19/94" & $9 / 9 V^{*}$ & $1910^{*}$ & $\mathrm{FV} / \mathrm{gV}$ & $9 \mathrm{~N} \cdot *$ & $\Delta / \backslash \varphi$ & $r / 9 \Delta$ & لاين هq \\
\hline $\mathrm{MN} \cdot{ }^{*}$ & $r / 9 \varphi^{*}$ & $4 \% *$ & $11 \% * *$ & $r_{\Delta} / .^{*}$ & $F T / *^{*}$ & $T / \mathscr{L}^{*}$ & $r / Q^{*}$ & لاين 99 \\
\hline$\Lambda r / \cdot *$ & $\mid F / r^{*}$ & $181 . *$ & $\mathrm{TV} / 0^{*}$ & $94 / 9 V^{*}$ & A./ & $\varphi / \cdot r^{*}$ & $T / \mu^{*}$ & لاين سب \\
\hline $9.1 *^{*}$ & 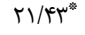 & 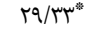 & "שr/" & $99 / \mu \Gamma^{*}$ & q./"ז & $r / 4 \varphi^{*}$ & $1 / M^{*}$ & لاين سر|| \\
\hline$M N \cdot{ }^{*}$ & $\Delta / V^{*}$ & $\mathrm{~W} / \mathrm{*}^{*}$ & $9 / \% T^{*}$ & $M F / \& V^{*}$ & $1.1 *^{*}$ & $\varphi / . \mu^{*}$ & $r / \mathrm{TV}$ & IR29 \\
\hline$\Lambda \mathrm{V} / \cdot$ & $11 / 1$ & 9.1. & $1 \% /$. & $\mid+1 /$. & $\mid K N$. & $\Delta / F^{Q} V$ & $r / \mathrm{TV}$ & $\begin{array}{r}\text { NONA BOKRA } \\
\text { (شاهد }\end{array}$ \\
\hline M/ " & $|F / \Lambda|^{*}$ & $r T / \mathscr{Q V} V^{*}$ & $\mathrm{~V} / \mathrm{0}^{*}$ & rN/gV & $11 \Delta /{ }^{\prime}$ & $\Delta / \Delta \Delta$ & $r / 9)^{*}$ & M614 \\
\hline M & $\mid F / \Delta F^{*}$ & $\mid r / \cdot *$ & $10 / .^{*}$ & $V T / \mu^{*}$ & $\Lambda \mu / *^{*}$ & $\Gamma / \Delta^{*}$ & $r / \Delta^{*}$ & M618 \\
\hline$N \varepsilon / 9 V^{\circ}$ & $1 . / \Delta T$ & $N / *^{*}$ & $\mathrm{rV} / \mathrm{*}^{*}$ & $\mathrm{rV} / \cdot *$ & $9 \% / *$ & $r / v \Lambda^{*}$ & Tش/א & M181 \\
\hline$M / \wedge 9^{*}$ & $r \cdot / \mu \varphi^{*}$ & $910^{*}$ & T\&/\%" & $v \varepsilon / *^{*}$ & $9 \% / 0^{*}$ & $F / \omega^{*}$ & $r / 99^{*}$ & M184 \\
\hline$M N / 9 V^{*}$ & $1 \% / 90$ & TE" & $r \Delta / / V^{*}$ & $V \Psi / \& V^{*}$ & $19 / 9 V^{*}$ & $r / \varphi^{*}$ & $\Gamma / T \Delta$ & M185 \\
\hline 19/\% " & $1 F / \pi F^{*}$ & " & $r \Delta / *^{*}$ & $1 . \Gamma / \pi T^{*}$ & $\Delta .1 *^{*}$ & $\varphi / \Lambda \Delta^{*}$ & תז/ץ & M436 \\
\hline / & ש & - & צ & $r / \cdot r \gamma$ & $1 / / \vee \wedge$ &.$/ D I T$ & $\cdot / \mu F$. & $\operatorname{LSD}(5 \%)$ \\
\hline
\end{tabular}




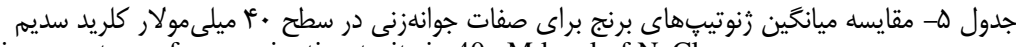
Table 5. Mean comparison of rice genotypes for germination traits in 40mM level of $\mathrm{NaCl}$

\begin{tabular}{|c|c|c|c|c|c|c|c|c|}
\hline درصد جوانهزنى & سرعت جوانهزنى & وزيشن خشهى & 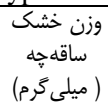 & 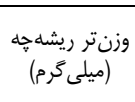 & وزنمتر ساقها & طول ريشهمتر) & 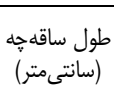 & \\
\hline $1.1 . *$ & $19 / \mathrm{V} \varphi^{\prime \prime \prime}$ & TQ/." & $r r / 9 V^{*}$ & $9 \Delta / 9 V^{*}$ & TTV/GV" & $F / \Delta)^{\prime \prime}$ & $F / / \mu^{* \prime \prime}$ & شصتك محمدى \\
\hline WV/rT & $r / 9 \cdot r^{*}$ & $10 / *^{*}$ & $19 / . *$ & 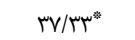 & $9 N / 9 V^{*}$ & $F /<8^{*}$ & $r / 4 Q$ & طارم جلودار \\
\hline ST/VN" & $1 . / \Lambda \mathrm{F}^{*}$ & $r /{ }^{*}$ & $r . \% *$ & $p q / . *$ & $\mathrm{Fr} / \mathrm{O}^{*}$ & $r / \omega \cdot *$ & $r / \cdot V$ & طارم محلى \\
\hline$\Delta F / \epsilon F^{*}$ & $r \cdot / r^{*}$ & $1910^{*}$ & $r .1 . *$ & $94 / . "$ & $19 / 9 V^{*}$ & $8 /$. & שT/T & لاين آ \\
\hline$\Lambda V / V \Lambda^{*}$ & $r . / q^{*}$ & $\mid f / r$ & $W / 9 V^{*}$ & $r \Delta / r^{\prime *}$ & $\mathrm{FV} / \mathrm{o}^{*}$ & $V / \omega \cdot *$ & $r / T r$ & لاين وه \\
\hline$\Delta \Delta / \Psi^{\prime *}$ & $r / N^{*}$ & $g / \mu^{*}$ & $\Delta / *^{*}$ & $\mathrm{rN} \cdot *$ & DI $/ .^{*}$ & $\mathrm{~V} / \mathrm{*}^{*}$ & $1 / r \cdot *$ & لاين و9 \\
\hline$V T / T^{*}$ & $\mid \omega / v \wedge$ & $10 / 9 V^{*}$ & 19/4" & gV/r"w" & $\| N / F V^{*}$ & $r / \Delta \mu^{*}$ & T/R & لاين سب \\
\hline$\Lambda \& / 1 *$ & $19 / 9 Y^{*}$ & $r \Delta / \& V^{*}$ & $\mid \mathrm{V} / \mathrm{*}^{*}$ & $1 . \Delta / \pi r^{*}$ & $1 . \Delta / *^{*}$ & $r / 4 \varepsilon^{* \prime}$ & $r / r$. & لاين سال \\
\hline$\Delta V / T T^{*}$ & $q / V e^{*}$ & $N / \Lambda r^{*}$ & $19 / 9 V^{*}$ & $r \varphi / 0^{*}$ & $\Delta V / q V^{*}$ & $\kappa / \mu^{*}$ & $1 / r^{m}$ & IR29 \\
\hline$V Q / \Delta \Delta$ & س & $\mid \% /$. & $r q /$. & $V \pi / \cdot$ & سז/ & $9 / 19$ & $r / T r$ & NONA BOKRA \\
\hline $90 / . *$ & $11 / r \Lambda^{*}$ & $1 \% \%$ & $\mathrm{re} / \mathrm{m}$ & D) $/{ }^{*}$ & $98 / 9 \mathrm{~V}^{*}$ & $\varepsilon / \lambda F^{*}$ & זיז/ז & M614 \\
\hline$V \& / g V^{*}$ & $|Q / F|$ & $10 / \mu^{*}$ & 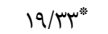 & 19/\%" & $9.10^{*}$ & F/ r r" & $r / T V$ & M618 \\
\hline$V \Psi / r^{*}$ & $1 \% / v \Lambda$ & $1 \% \%$ & $\mid \mathrm{V} / \mathrm{*}^{*}$ & 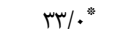 & $9 / / 9 V^{*}$ & $r / 4 \varepsilon^{*}$ & $r / \mu \cdot *$ & M181 \\
\hline $99 / 11 \%$ & $\mid F / 1 \cdot 0^{*}$ & $\mid \omega / \Gamma T^{*}$ & $r \Delta / . *$ & $1 . .10^{*}$ & 1.V/r" & $1 / F a^{*}$ & $r / \uparrow \varphi^{*}$ & M184 \\
\hline $99 / 4 \varphi^{*}$ & $I T / r \theta^{*}$ & $\mid \mathrm{V} / 0^{*}$ & $\mid r / q V^{*}$ & $1 \% \% *$ & $11.19 \mathrm{~V}^{*}$ & $r / F T^{*}$ & $r / q G$ & M185 \\
\hline $9.10^{*}$ & $1 . / \mu^{m}$ & $19 / \Gamma^{*}$ & 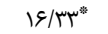 & $r \Delta / *^{*}$ & $1.9 / 9 \mathrm{~V}^{*}$ & $\Delta / \Gamma \Lambda^{*}$ & T/q & M436 \\
\hline $1 / \mu^{\prime}$ & $1 / \cdot r$ & ז"זא/. & $1 / 4$ & $t / \cdot t^{\prime}$ & $F / \Lambda$ & $\cdot / 0$ & ( & $\operatorname{LSD}(5 \%)$ \\
\hline
\end{tabular}

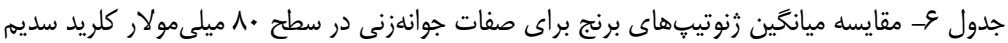
Table 6. Mean comparison of rice genotypes for germination traits in $80 \mathrm{mM}$ level of NaCi

\begin{tabular}{|c|c|c|c|c|c|c|c|c|}
\hline جوانهزنى درصد & جوانهزنى & وزن خشهُ & 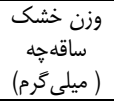 & 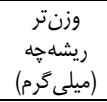 & وزنتر ساقه & طول ريشهمتر) & طول ساقه خه & صفات \\
\hline$V T / T^{*}$ & $1 \% / 9 \cdot 1^{*}$ & $19 / . *$ & 每 & $9 \mathrm{~V} / 0^{*}$ & $\Lambda r / *^{*}$ & $f / T^{\prime \prime}$ & $r / \omega^{*}$ & شصتك محمدى \\
\hline$\Delta F / . *$ &.$/ 1 r D^{*}$ & $s / r^{*}$ & $\mathrm{~V} / 9 \mathrm{~V}^{*}$ & $\mid V / r T^{*}$ & 每 & ५/৭"* & $1 / v$ & طارم جلودار \\
\hline $90 / . *$ & $11 / 4$ & $181 . *$ & rQ/r"r: & $\Lambda \mathrm{V} / \mathrm{*}^{*}$ & $\mid r F / g V^{*}$ & $r / \Delta)$ & 海 & طارم محلى \\
\hline$\Lambda T / Y \Lambda^{*}$ & $M / \cdot r^{*}$ & $\mathrm{~N} / *$ & $\mid r / V^{*}$ & $T V / . *$ & $g V / \mu^{*}$ & $r / \Lambda$ & $1 / \mu r$ & لاين Tr \\
\hline $99 / 44^{*}$ & IT/K & $1 \% \% *$ & $1 \% / . *$ & $94 \% *$ & $F N / \mu^{*}$ & $\mathrm{r} / \mathrm{\Lambda}^{*}$ & 1/ז & 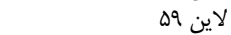 \\
\hline rq/." & $r / N^{*}$ & $F / *$ & $8 /$. & $r M / * *$ & $F / *$ & $r / . r$ & $1 / \cdot e^{*}$ & لاين 999 \\
\hline$\Lambda \cdot / \Delta \Delta^{*}$ & $|\varepsilon / \Lambda|^{* 3}$ & $N \cdot * *$ & $\mid r / r^{*}$ & $<q / *^{*}$ & $\Delta F / T^{*}$ & $F / \Delta^{*}$ & $1 / M^{*}$ & لاين سس \\
\hline$V \Delta / \Delta \Delta^{*}$ & $|Q / \mathcal{A}|^{*}$ & $\mathrm{IV} / \cdot^{*}$ & $10 / *^{*}$ & $\mathrm{rN} \cdot{ }^{*}$ & $\mid r \Delta / \cdot *$ & r T/g & $1 / \Delta S$ & لاين سال \\
\hline $\mathrm{F} \mid \mathrm{V} \Lambda^{*}$ & $s / R v^{*}$ & $r / *^{*}$ & $T / \kappa^{*}$ & $\mathrm{~N} / *$ & $\mid r / . *$ & $1 / N{ }^{*}$ & . $/ \Lambda r^{*}$ & IR29 \\
\hline $94 / 44$ & $11 / 1$ & s/. & $\Delta / V$ & $\Delta \mathrm{V} / \mathrm{T}$ & $T V / V$ & ז/rq & $1 / \Delta 1$ & NONA BOKRA \\
\hline SV/TY" & $11 / 94$ & $\mathrm{~V} / *^{*}$ & $1 / * *$ & $11 \% * *$ & $\mathrm{fV} / \mathrm{r}^{*}$ & $r / \mu^{*}$ & $1 / \uparrow \Delta^{*}$ & M614 \\
\hline$G T / T T^{*}$ & $11 / . r$ & $\Delta / r^{*}$ & $\mid \mathrm{V} / \mathrm{*}^{*}$ & $r q / V^{*}$ & $\Delta \mathrm{V} / *^{*}$ & $r / M *$ & $T / T Q^{*}$ & M618 \\
\hline$s T / V \Lambda^{*}$ & $1 \cdot / v$ & $\Delta / * *$ & १/** & $11 / * *$ & $r V / \mu^{*}$ & $r / \& \Lambda^{*}$ & $r / 19^{*}$ & M181 \\
\hline$\Delta T / T^{*}$ & $8 / 90^{* \prime}$ & $\Delta / *^{*}$ & $|\varepsilon| \cdot{ }^{*}$ & $\mathrm{rN} \cdot *$ & $\Delta / *^{*}$ & $F(\Delta)^{*}$ & $r / N^{*}$ & M184 \\
\hline$\Delta T / T T^{*}$ & $N / \Delta H^{*}$ & $\%$ * & $V / \mathrm{r}^{*}$ & $9 \Delta /{ }^{*} *$ & $\Delta \mathrm{N} / \mathrm{K}^{*}$ & $1 / 9 f^{*}$ & "T/K" & M185 \\
\hline SV/TY" & $\| / \wedge \Delta$ & $\mathrm{N} \cdot{ }^{*}$ & $\mid f /$. & $\Delta / \cdot *$ & $\Delta \Delta / *^{*}$ & $r / \cdot V^{*}$ & ו/r & M436 \\
\hline $1 / 494$ & $1 / \cdot r$ &.$/ \$ \Delta$ & 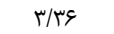 & 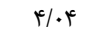 & $r / M V$ &.$/ 49$ &.$/ \pi$ & $\operatorname{LSD}(5 \%)$ \\
\hline
\end{tabular}


جدول V- مقايسه ميانگين زنوتيِهاى برنج براى صفات جوانهزنى در سطح • rا ميلى مولار كلريد سديم Table 7. Mean comparison of rice genotypes for germination traits in $120 \mathrm{mM}$ level of $\mathrm{NaCl}$

\begin{tabular}{|c|c|c|c|c|c|c|c|c|}
\hline 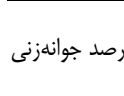 & رعت جوانهز & 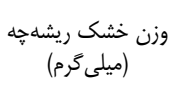 & وزاقن خشك & 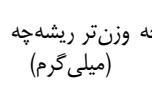 & وزنتر ساقه هِ & طول ريشه & صفات طول ساقه خه & \\
\hline $\mathrm{V} / / \mathrm{V}^{*}$ & $\mid T / V e^{*}$ & $8 / \pi \Psi^{*}$ & $1 N / 9 V^{\prime \prime}$ & $F Y / *^{*}$ & $90 / T^{*}$ & $T / V \Delta$ & $1 / \% 0^{*}$ & شصتك محمدى \\
\hline$F Y / Q V^{*}$ & $N / 19^{*}$ & 1/r" & 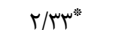 & $81 . *$ & $\mid Q / \mu^{*}$ & $r / \mu I^{*}$ &.$/ \Delta V^{*}$ & طارم جلودار \\
\hline $9.10^{*}$ & $1 . / 19$ & $\cdot / 1^{*}$ & $g / T^{\prime \prime}$ & $r / *^{*}$ & $1810^{*}$ & $1 / \Delta \Lambda^{*}$ &.$/ 9 \Lambda^{*}$ & طارم محلى \\
\hline$V \Gamma / \Lambda^{*}$ & $10 / N^{*}$ & $\mathrm{~V} / \mathrm{*}^{*}$ & $910^{*}$ & $\mu \varphi / . *$ & $\tilde{F V} / \mathrm{r}^{*}$ & $r / \cdot \varphi^{* \prime}$ & $1 / \cdot \Delta \mu^{*}$ & لاين Ir \\
\hline$\Delta \Delta / \Delta \Delta^{*}$ & $q / r \varepsilon$ & $810^{*}$ & $s / w^{*}$ & $41 / *$ & $r \Delta / r^{*}$ & $\Delta / .^{*}$ & $1 . \cdot 9 r^{*}$ & لاين \\
\hline "ע/או" & N/W" &.$/ 1^{*}$ &.$/{ }^{*}$ & $1 / *^{*}$ & $r / V^{*}$ & $\cdot / \Lambda \propto V^{*}$ & 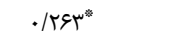 & لاين 99 \\
\hline$\Delta N / \mu^{*}$ & $1 . / T \Lambda$ &.$/ 1^{*}$ & $\Delta / *^{*}$ & $r) / *$ & r $/ . *$ & $r / r \Lambda$ & $\cdot / \Lambda r^{*}$ & لاين ست \\
\hline$V T / T T^{*}$ & $1 \% / 10^{*}$ & 91." & $\Delta / *^{*}$ & $F / *$ & $\mathrm{rV} / 0^{*}$ & $r / \Delta T^{*}$ & $\cdot / V T^{*}$ & لاين سا| \\
\hline rm/ & $r / \mu^{*}$ & $\cdot /^{*}$ & $\cdot 1^{*}$ & $1 / *$ & $r / v^{*}$ & ./А१४" &.$/ q^{*}$ & IR29 \\
\hline$\Delta r / \wedge q$ & $q / \cdot{ }^{f}$ & $\%$ & $\% /$ & $\mathrm{V} / \cdot$ & $\mathrm{V} / \cdot$ & $r / 1$ & $r /$. & NONA BOKRA \\
\hline$\Delta .1 *^{*}$ & $T / \Lambda^{*}$ & $r / \mu^{*}$ &.$/ \%$ & $1 / *$ & $1 / \mu^{*}$ &.$/ V V r^{*}$ & $\cdot / r \cdot V^{*}$ & M614 \\
\hline TH/TY" & $\Delta / V)^{*}$ & $\cdot 11^{*}$ & $r /$. & $r / *$ & $\Lambda / \sim^{*}$ & $1 / 41$ &.$/ 19 v^{*}$ & M618 \\
\hline $\mathrm{rV} / \mathrm{V} \Lambda^{*}$ & $81 \cdot V^{*}$ & $.11^{*}$ & $.1^{*}$ & $\mathrm{~N} \cdot *$ & 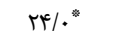 & $r / \cdot V^{*}$ & $\cdot / V^{*}$ & M181 \\
\hline $\mathrm{rr} / \mathrm{*}^{*}$ & $F / \notin \varepsilon^{*}$ & $\%$ & $\mid r / \mu^{*}$ & $1 \% / \cdot *$ & $\Delta / \cdot *$ & $r / V T$ & $1 / 4 \varphi^{*}$ & M184 \\
\hline$\Delta Q / \Delta Q^{*}$ & $9 / \cdot 1$ & $\cdot / 1^{*}$ & $\cdot 1^{*}$ & $\mathrm{IV} / \mathrm{*}^{*}$ & $\mathrm{~V} /$. & $M / \Lambda^{*}$ &.$/ \$ \Delta^{*}$ & M185 \\
\hline ( & $1 . / 99$ & $81 *^{*}$ & $ץ /$ & $1 Q / \mu^{*}$ & $\mathrm{~N} / *^{*}$ & $r / 19$ &.$/ \mathrm{VAV}^{*}$ & M436 \\
\hline T/FT &.$/ \Lambda$ & . IQT & $1 / T$ & .191 & $1 / 1 \vee \wedge$ &.$/ Q T$ &.$/ \mu$ & $\operatorname{LSD}(5 \%)$ \\
\hline
\end{tabular}

خشك ساقهجه كه با صفت درصد جوانهزنى رابطه مثبت

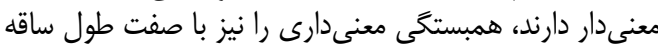

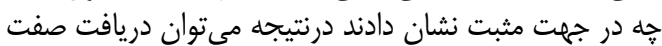

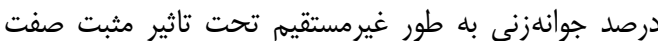

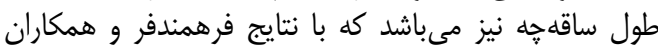

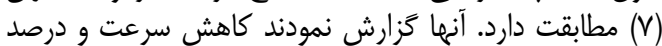

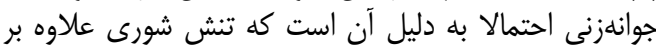

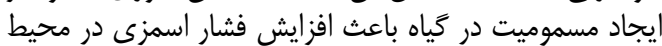

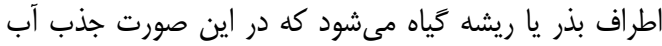

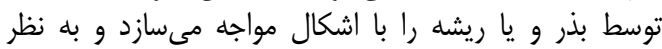

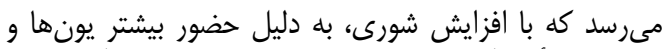

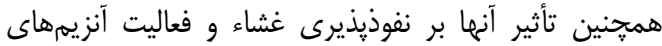

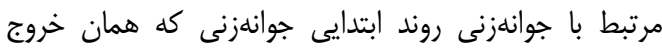
ريشه ֶِه باشد با سرعت بيشترى انجام شده است.
ضرايب همبستخى ساده ضرايب همبستخى بين صمبست ساده صنات مورد مطالعه نشان داد

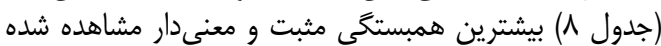

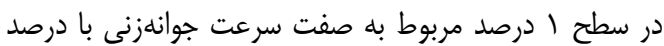

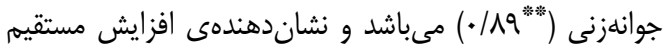

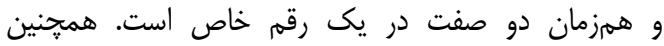

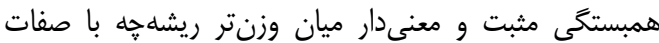
درصد جوانهزنى (

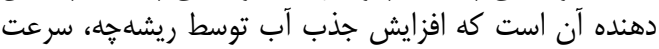

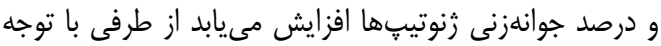

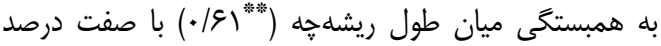

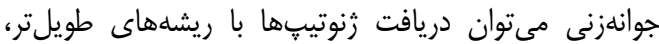

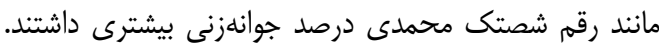

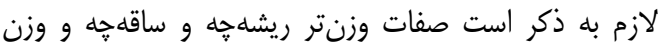

Table 8. Simple correlation coefficients of evaluated traits

\begin{tabular}{|c|c|c|c|c|c|c|c|c|}
\hline جوانهزنى درصد & جوانهزنى & وزن خشه & وزن خشهى & روزن تر & ساقه & ريشهإه & ساقه & صفات \\
\hline & & & & & & & 1 & 1 \\
\hline & & & & & & 1 &.$/ 4 V$ & r \\
\hline & & & & & 1 & $\cdot / f$ & $\cdot / V^{m} * *$ & r \\
\hline & & & & 1 & $\cdot \mid \& \wedge * *$ &.$/ 4 V$ & $\cdot|\Delta|^{*} *$ & f \\
\hline & & & 1 & ./V9** & - / 1 १** & $\cdot / \& \wedge$ & ./Vq** & $\Delta$ \\
\hline & & 1 & rT/. & $\cdot / \Delta F^{*}$ & $\cdot|\varepsilon| *$ & - $/ \Delta T^{*}$ & ./\%q & 9 \\
\hline & 1 &.$/ 19 q$ & $\cdot / \Delta \gamma^{*}$ & $\cdot / V r^{* * *}$ & $\cdot / \Delta F^{*}$ & $\cdot / \& V$ & . & v \\
\hline 1 & - $/ 19 * *$ &.$/ 09 *$ & $\cdot \mid \Delta \xi^{*}$ & $\cdot / V^{* * *}$ & $\cdot / \Delta \Lambda^{*} *$ & $\cdot|9| *$ & . & $\wedge$ \\
\hline
\end{tabular}

سرعت جوانهزنى در جهت مثبت است به طورى كو كه در

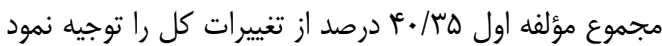

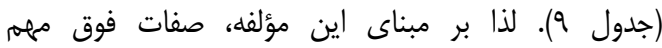

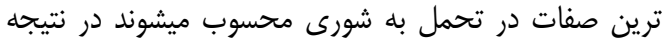

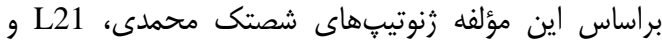

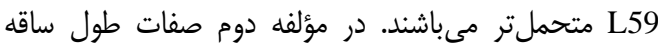

تجزيه به مؤلفههاى اصلى

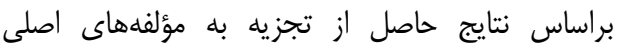
براساس هشت صفت در مرحله جوانهزنى نشان داد داد كه تعداد

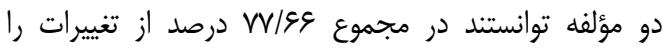

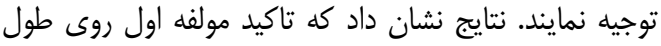

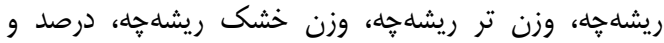


نمايش باىيلات زنوتيههاى برنج مورد مطالعه بر اساس

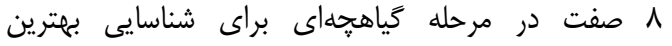

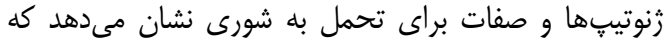

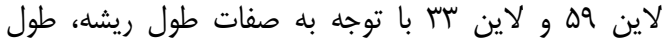

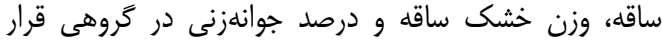

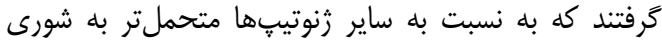

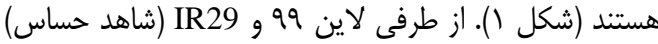

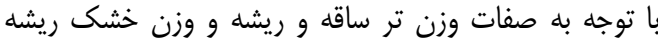
نسبت به ساير زنوتيڤها حساستر نسبت به شورى مى باشيد.

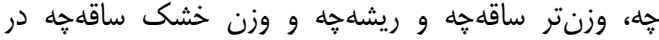

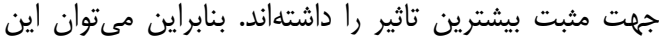

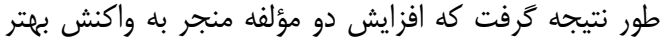

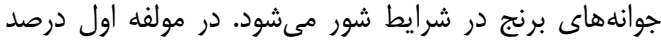

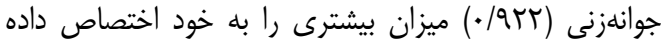

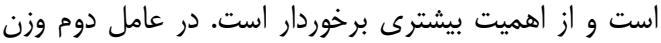

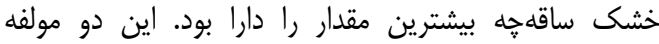

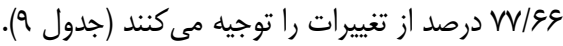
نمايش باى يلات

Table 9. Principle component analysis for evaluated traits

جدول q- تجزيه به مؤلفهها اصلى براى صفات مورد مطالعه

\begin{tabular}{|c|c|c|}
\hline 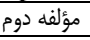 & مؤلفه اول & صفات \\
\hline ./MA & - ISA & طول ساقه \\
\hline . /TAL & - $\mid \& \Delta \Delta$ & طول ريشهِه (سانتىمتر) \\
\hline - /AFS & . & وزن تر ساقه ֶֶ (ميلى گرم) \\
\hline . & 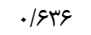 & وزن تر ريشه \\
\hline$. / 9 .$. & וזس/. & وزن خشك ساقه \\
\hline$\cdot /$ TAI & . MIT & وزن خشك ريشه ֶֶ (ميلى \\
\hline.$/ 499$ & - /AFs & سرعت جوانهزنى س \\
\hline 2 & . $/ 94 \pi$ & درصد جوانهزنى (\%) \\
\hline $1 / 9$ & $T / V$ & مقادير ويثه \\
\hline $\mathrm{TV} / \mathrm{T} \cdot 1$ & $f \cdot / \pi \Delta$ & واريانس نسبى (\%) \\
\hline$V / / 991$ & $\tau \cdot / \pi \Delta$ & واريانس تجمعى (٪) \\
\hline
\end{tabular}

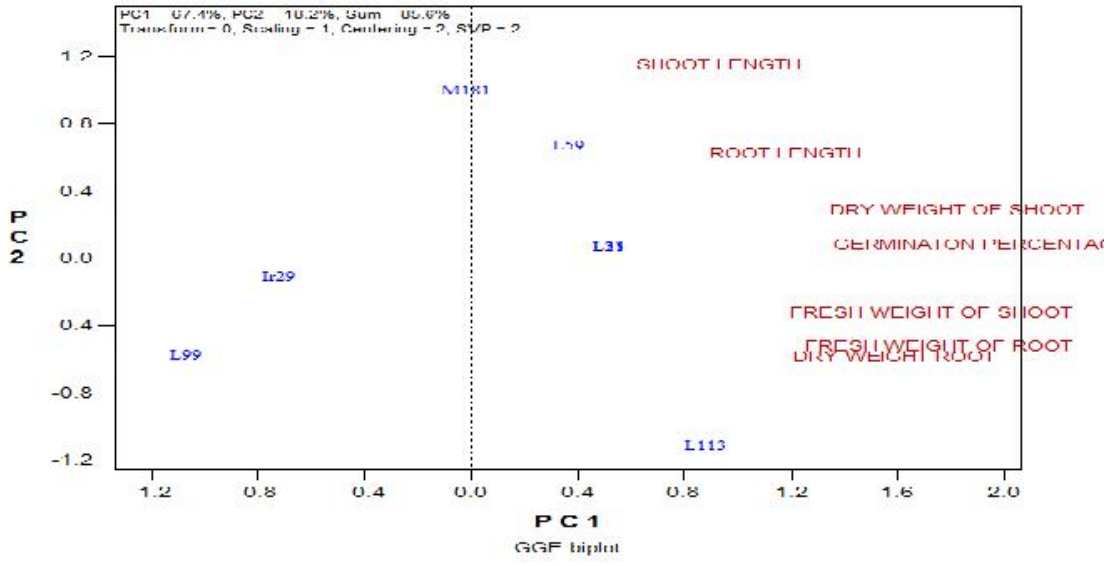

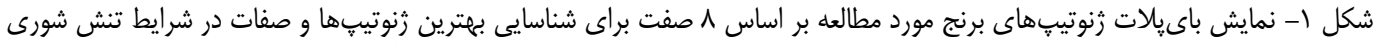
Figure 1. Showing the biplot of rice genotypes based on 8 traits to identify the best genotype (s) and traits in salt stress conditions

دادهاى بدست آمده (جدول r) كروه سوم تحمل به شورى

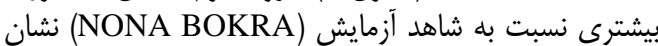
دادند.

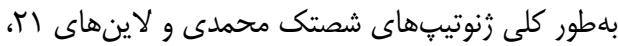

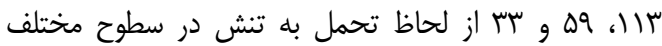

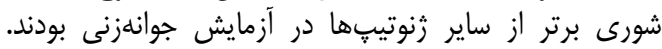

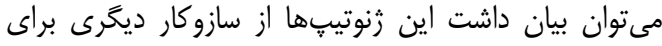

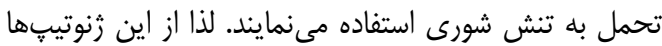
مى توان در ساير برنامههاى اصلاحى استفاده نمود.
تجزيه خوشهاي

دندروگرام تجزيه خوشهاى ارقام تحت تراي تنش سطوح

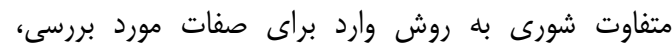

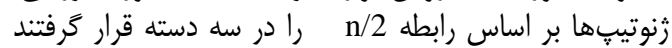

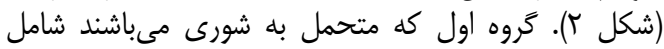
زنوتيّهاى NONA BOKRA،

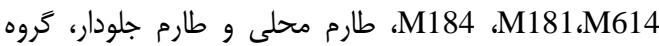
دوم شامل زنوتيبهاى L99 و

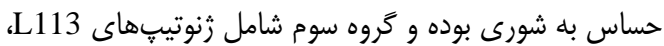

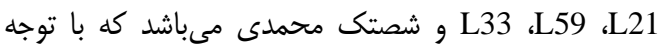




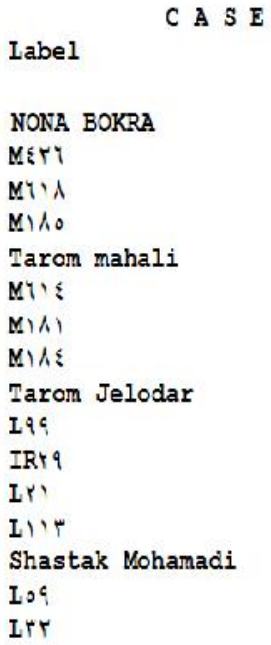

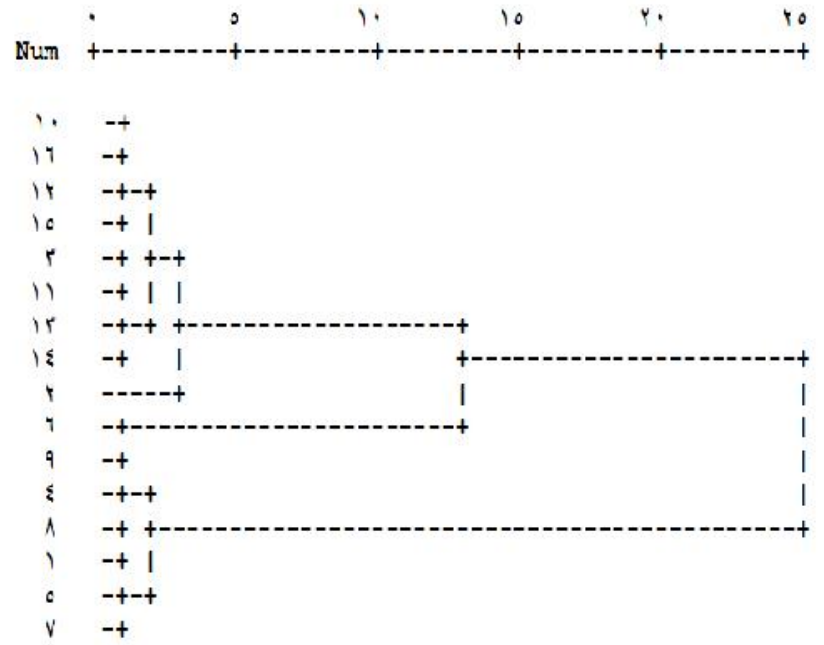

شكل r- دندروگرام خوشاه زنوتيڤهاى برنج مورد مطالعه تحت تنش شورى

Figure2. Cluster analysis study on rice genotypes under salt stress

1. Abbas, M.K., S.A. Ali, H.H. Hasan and R.H Ghal. 2013. Salt tolerance study of six cultivars of rice (Oryza sativa L.) during germination and early seedling growth. Journal of agricultural science .Vol. 5, No. 1; ISSN, 250-259 pp.

2. Anbumalarmathi1, J. and P. Mehta. 2013. Effect of salt stress on germination of indica Rice varieties. EJBS, 6(1): 1-6.

3. Ashrafi, S.H., R. Hoseini, G.H. Garusi, A. Hadad and V. Morad nezhad. 2013. Nuclease enzyme activity rather than changes in the DNA single-strand braid, protein and chlorophyll a sensitive and tolerant to salt stress in two varieties of barley. Journal of cells and tissues, 2(4): 178-195 (In Persian).

4. Dubey, Y. and M. Rani. 1998. Influence of $\mathrm{NaCl}$ salinity on growth and metabolic status of protein and amino acids in rice seedlings. Department of biochermstijy faculty of science, Banaras. Hindu university varanasi-221005, India. J. Agronomy \& Crop Science, 162: 97-106.

5. Eizad dost, H., H.A. Sami zade, B. Rabiei and S.H. Abdolahi. 2013. Evaluation of salinity tolerance in rice cultivars (Oryza sativa L.) with emphasis on stress tolerance index. Journal of cereal research, 3(3): 167-180 (In Persian).

6. Falah, A., E. Farahmand far and F. Moradi. 2015. Effect of salinity in different growth stages on some physiological characteristics of rice varieties in greenhouse. Journal of Agriculture, 107: 175-182 (In Persian).

7. Farahmand far, E., K. Postitni, A. Falah, R. Tavkol afshari and F. Moradi. 2009. Effect of salt stress on germination and early growth of rice cultivars and genotypes (Oryza sativa L.). Iranian Journal of Field Crop Science, 3(40): 71-94 (In Persian).

8. Ghasem khani, M. and G.H. Mohamadi nezhad. 2012. Locating genetic traits associated with salinity tolerance during germination and growth of rice. Journal of Agricultural Biotechnology, 2(4): 43-59 (In Persian).

9. Gholizade, F., S. Navab pur, H. Sabori and S.S. Ramezan pur. 2013. The effects of salinity on growth and physiological characteristics of rice genotypes at seedling stage in hydroponic. Journal of the crop, 1(5): 79-92 (In Persian).

10. Gholizade, F. 2012. The effect of salinity on seed germination of rice genotypes (Oryza sativa L.). Journal of Cell-Molecular Biotechnology News, 6(2) 1-7 (In Persian).

11. Ghomi, K.H. Sabori, H. Rabiei and A. Sabori. 2013. Evaluation of Seedling Stage and identify appropriate criteria to choose a segregating population salinity in rice (Oryza sativa L.). Journal of crop breeding, 12(5): 30-38 (In Persian).

12. Ghorbani, M.H., T.A. Hoseini and M. Zahed. 2007. Ten varieties of rice vegetative growth response to salt stress. A visa Agriculture and plant breeding, 1-8 (In Persian).

13. Habibolahi, N., M. Mahdie and M.R. Amirjani. 2012. The effect of salinity on growth, proline, the activity of antioxidant enzymes and efficiency of photosystem II in resistant rice varieties. Journal of biotech crops, 1(1): 1-11 (In Persian).

14. Hakim, M.A., A.S. Juraimi, M. Begum, M.M. Hanafi, R. Ismail Mohd and A. Selamat. 2010. Effect of salt stress on germination and early seedling growth of rice (Oryza sativa L.). African journal of biotechnology, 9(13): 1911-1918.

15. Hakim, M.A., A.S. Juraimi, M.M. Hanafi1, M.R. Ismail, M.Y. Rafii, M.M. Islam and A. Selamat. 2014. The effect of salinity on growth, ion accumulation and yield of rice varieties. The Journal of animal and plant Sciences, 24(3): 874-885. 
16. Islam, M.M. and M.A. Karim. 2010. Evaluation of rice (Oryza sativa L.) genotypes at germination and early seedling stage for their tolerance to salinity. A scientific journal of Krishi foundation. The agriculturists, $8(2):$ 57-65.

17. Mohamad zade, M., M. Noruzi, S.A. Peyghambari and A.R. Nabi Pur. 2009. Evluation of rice genotypes to salinity stress at germination stage. Journal of crop breeding, 1(1): 10-21 (In Persian).

18. Moradi nezhad, S.H. and M. Vazir Pur. 2007. Assessment of changes in viability, proline and chlorophyll local rice genotypes under salt stress. Journal of new agricultural science, 8(3): 69-80 (In Persian).

19. Ologundudu, A.F., A.A. Adelusi and R.O. Akinwale. 2014. Effect of salt stress on germination and growth parameters of rice (Oryza sativa L.). Not Sci Biol. Print ISSN, 237-243.

20. Rezaeian, M., M.A. Faramarzi, M. Niknam and H. Ebrahimi. 2014. The effect of salinity on growth, lipid peroxidation and phycobiliproteins Padaksayndh industries of the two species NostOk. Journal of Plant Research (Iranian Journal of Biology), 4(27): 661-673 (In Persian).

21. Sharifi, P. 2013. Evaluation of Salinity on the number of traits in rice seed germination. Journal of Plant and Ecosystem, 9: 1-34 (In Persian). 


\title{
Evaluation of Reaction of some Rice Genotypes to Salinity Stress at Germination Stage
}

\author{
Seyede Fateme Mohamadi ${ }^{1}$, Nadali Bagheri ${ }^{2}$, Ghafar Kiani ${ }^{3}$ and Nadali Babaeian Jelodar ${ }^{4}$
}

1, 3 and 4-Graduted M.Sc. Student, Assistant Professor and Professor, Department of Plant Breeding, of Sari Agricultural Sciences and Natural Resources University,

2- Assistant Professor Department of Plant Breeding, of Sari Agricultural Sciences and Natural Resources University (Corresponding author: n.bagheri@sanru.ac.ir)

Received: January 24, $2017 \quad$ Accepted: April 3, 2018

\begin{abstract}
In order to evaluate the response of rice genotypes to salinity stress at germination stage an experiment was conducted as a factorial experiment in a completely randomized design with three replications in Biotechnology La of Sari Agricultural Sciences and Natural Resources University (SANRU). Sixteen rice genotypes were evaluated at four salinity levels $(0,40,80$ and $120 \mathrm{mM}$ ). The results of variance analysis showed a significant effect of salinity on the traits in different rice genotypes. According to the results of the mean comparison of the studied traits, with increasing salinity from the control level to 40,80 and $120 \mathrm{mM}$, all traits showed a significant decrease. A simple correlation coefficient between the studied traits showed that the highest positive and significant correlation was observed between germination rate with germination percentage $(\mathrm{r}=0.89)$. The results of the principal component analysis based on the eight traits in the germination stage showed that the two components were able to justify $77.66 \%$ of total changes. The first component emphasized on root length, root fresh weight, root dry weight, percentage and germination rate in positive direction. The cluster analysis of rice genotypes under stress of different levels of salinity on the basis of studied traits showed that L113, L21, L59, L33 and Shasttak Mohammadi genotypes in the third group had more salt tolerance than control (NONA BOKRA). Regarding the analysis, Shultak Mohammadi and L21 genotypes in terms of tolerance to stress in different levels of salinity were superior to other genotypes in this experiment and could be used in breeding programs.
\end{abstract}

Keywords: Cluster analysis, Germination, Principle component analysis, Rice, Salinity 